\title{
Technical Note: Calibration device for the krypton hygrometer KH20
}

\author{
T. Foken ${ }^{1,3}$ and H. Falke ${ }^{2}$ \\ ${ }^{1}$ University of Bayreuth, Department of Micrometeorology, Bayreuth, Germany \\ ${ }^{2}$ Gesellschaft für Akustik und Fahrzeugmesswesen mbH, Zwickau, Germany \\ ${ }^{3}$ Member of Bayreuth Center of Ecology and Environmental Research (BayCEER), University of Bayreuth, \\ Bayreuth, Germany
}

Correspondence to: T. Foken (thomas.foken@uni-bayreuth.de)

Received: 27 January 2012 - Published in Atmos. Meas. Tech. Discuss.: 17 February 2012

Revised: 3 June 2012 - Accepted: 3 July 2012 - Published: 1 August 2012

\begin{abstract}
A calibration device for krypton hygrometers (KH20, Campbell Scientific, Inc.) with variable path length is presented. This unit allows for in-situ calibrations of the krypton hygrometer, which is typically not very stable over time, during measuring campaigns. It was constructed mainly for application at high altitudes and low temperatures, where further improvements are needed to the IRhygrometers which are normally used. The changing path length requires that a changing concentration of the absorber be simulated. Because oxygen absorbs more strongly than water vapour, the calibration is made against oxygen and transferred to water vapour. The design of the calibration instrument is made as one unit containing a stepper motor system, PC and humidity sensor. For the calibration, it is necessary to install the krypton hygrometer on this unit.
\end{abstract}

\section{Introduction}

Fast response hygrometers are nowadays an important part of all measuring complexes for turbulent energy fluxes. There has been a significant change in the measuring systems within the last $10-15 \mathrm{yr}$. While sensors which were commercially available in the 90s of the previous century mainly used UV absorption lines (Foken et al., 1995), at present there is almost exclusive use of sensors working with the IR absorption lines. This is because the hydrogen lamp for hygrometers working at the Lyman-alpha line of $121.56 \mathrm{~nm}$ is not very stable, and the lamps were mainly handmade. At the same time, the sensitivity of IR-sensors has been increased and while these sensors have only been commercially available since the 90s, the first developments of devices are dated $30 \mathrm{yr}$ earlier (for overview see Foken et al., 1995).

The Lyman-alpha hygrometer was developed at the beginning of the 1970s almost in parallel in the USA, the Soviet Union and the former GDR (Buck, 1973; Kretschmer and Karpovitsch, 1973; Martini et al., 1973) and the American device was commercially produced by AIR Inc., Boulder, $\mathrm{CO}$. About ten years later a second type of UV hygrometer was developed using a krypton lamp (Campbell and Tanner, 1985). The benefits of this device were a longer lifetime and easier production. But the absorption band is not directly located in the Lyman-alpha band and has a cross sensitivity to oxygen (see Sect. 2).

All these hygrometer types work on the basis of LambertBeer's law

$I=I_{0} \exp \left(-\rho_{\mathrm{w}} k x\right)$

with the light intensity at the receiver $I$ and of the lamp $I_{0}$, the absolute humidity $\rho_{\mathrm{w}}$, the absorption coefficient $k$ and the path length between lamp and receiver $x$. While emission and detection efficiencies affect $I_{0}$, these do not affect flux measurements since only the fluctuation level needs to be determined. However, knowledge of $k$ and $x$ is required for proper scaling of fluctuations in $\rho_{\mathrm{w}}$.

The calibration procedure has usually been carried out for different absolute humidities at fixed path lengths. Because the hydrogen and the krypton lamps are not very stable in time and the calibrations in a moisture chamber are not very practical during field campaigns (time consuming and often 
such chambers are not available), the calibration with variable path length was developed. According to Eq. (1) the calibration with a changing path length is also possible in the case of a constant absolute humidity. A first Lymanalpha hygrometer with variable path length was proposed by Buck (1976) and an updated version by Foken et al. (1998). A simple application of this system to the krypton hygrometer is impossible, because this device works with two absorption lines, each with two absorbers. Nevertheless, a development is necessary because IR hygrometers are not very sensitive in measuring absolute humidities below $2-4 \mathrm{~g} \mathrm{~m}^{-3}$, and in this range the calibration is not very linear unless a special calibration for these conditions is applied, which is - under complicated environmental conditions - difficult to realize. But such low absolute humidities are typical in cold regions and at high altitudes. Therefore, a calibration system with variable path length for krypton hygrometers was developed and is presented in this paper.

\section{Theory of the krypton-hygrometer and the calibration device}

\subsection{Characteristics of the krypton lamp}

The source of the krypton hygrometer $\mathrm{KH} 20$ is a lowpressure krypton glow tube. Emission from the krypton tube exhibits a minor band at $116.49 \mathrm{~nm}$ (band 1) and a major band at $123.58 \mathrm{~nm}$ (band 2). Radiation at $123.58 \mathrm{~nm}$ is strongly attenuated by water vapour, whereas absorption by other gases in the optical path is relatively weak at this wavelength. Radiation at the shorter wavelength $(116.49 \mathrm{~nm})$ is attenuated by water vapour and also by oxygen molecules, but the intensity of the transmitted beam is considerably reduced at this wavelength by magnesium fluoride windows fitted to the source and detector tubes (Campbell and Tanner, 1985). The spectrum of the krypton lamp is shown in Fig. 1.

Therefore, the output signal depends on both path lengths and both absorbers: water vapour and oxygen (Buck, 1976; Campbell and Tanner, 1985)

$$
\begin{aligned}
I & =I_{01} \exp \left[-x\left(k_{\mathrm{w} 1} \rho_{\mathrm{w} 1}+k_{\mathrm{o} 1} \rho_{\mathrm{o} 1}\right)\right] \\
& +I_{02} \exp \left[-x\left(k_{\mathrm{w} 2} \rho_{\mathrm{w} 2}+k_{\mathrm{o} 2} \rho_{\mathrm{o} 2}\right)\right]
\end{aligned}
$$

where the indices 1 and 2 refer to the path length for $116.49 \mathrm{~nm}$ and $123.58 \mathrm{~nm}$, respectively, and the indices $\mathrm{w}$ and $o$ for water vapour and oxygen.

According to Tillman (1965) the absorption coefficient in the short wavelength is half of that in the longer wavelength. Because of this, and because only the signal of these two wavelengths is measured, it is possible to combine both water vapour absorptions. For the oxygen absorption the fraction of band 1 is $f$ and of band 2 is $(1-f)$. The simplified Eq. (2) is

$$
\begin{aligned}
I & =I_{0} \exp \left(-x k_{\mathrm{w}} \rho_{\mathrm{W}}\right)\left[f \exp \left(-x k_{\mathrm{o} 1} \rho_{\mathrm{o} 1}\right)\right. \\
& \left.+(1-f) \exp \left(-x k_{\mathrm{o} 2} \rho_{\mathrm{o} 2}\right)\right] .
\end{aligned}
$$

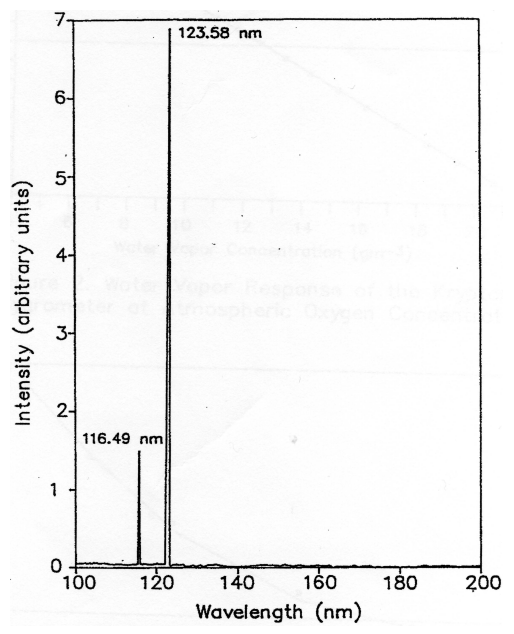

Fig. 1. Spectrum of the krypton lamp (Campbell and Tanner, 1985, courtesy Scientific Services, Rocky Hill, NJ).

For most applications, the additional oxygen absorption can be ignored and the basic equation for the calibration of the Campbell Scientific, Inc. krypton hygrometer KH2O is

$\ln (V)=\ln \left(V_{0}\right)-a \cdot x \cdot K_{\mathrm{w}}$

with the constant $V_{0}$ in $\ln (\mathrm{mV})$, approximately $8 \ldots 10$ $\ln (\mathrm{mV})$, the intercept $\exp \left(\ln V_{0}\right)$ in $\mathrm{mV}$, the calibration coefficient (effective absorption coefficient for water vapour) $K_{\mathrm{w}}$ in $\ln (\mathrm{mV}) \mathrm{m}^{3} \mathrm{~g}^{-1} \mathrm{~cm}^{-1}$, the coefficient $x K_{\mathrm{w}}$ in $\ln (\mathrm{mV}) \mathrm{m}^{3} \mathrm{~g}^{-1}$ for a given path length $x$ in cm after this simplification and the absolute humidity $a$ in $\mathrm{g} \mathrm{m}^{-3}$.

This simplification is possible because only the measurement of the fluctuations of the absolute humidity is of interest. If the oxygen concentration does not change (for nearly constant air pressure), this calibration can be used. Nevertheless small corrections are recommended (Tanner et al., 1993; van Dijk et al., 2003).

\subsection{Calibration with variable path length}

Since a change in the output signal can be produced by either a change in humidity and oxygen or a change in path length, it is possible to alter the path length for nearly constant humidity and oxygen conditions in order to simulate a change in humidity or oxygen. This makes a calibration with a variable path length for constant humidity possible for the Lyman-alpha-hygrometer (Foken et al., 1998), because the ratio of the absorption coefficients is about $2 \times 10^{3}$ (Tillman, 1965), while the ratio of the densities is typically about $2 \times 10^{-1}$ and therefore the effect of the water vapour is 100 fold higher than that of the oxygen.

This is not the case for the krypton hygrometer, where the absorption coefficients of water vapour and oxygen are of the same order of magnitude when the partial density of oxygen is at least twentyfold higher. The absorption coefficient for water vapour used in the krypton hygrometer 
papers were referred to in Watanabe and Zelikoff (1953) and Johns (1965). According to the more recent paper by Mota et al. (2005) ${ }^{1}$ with data of a spectral solution of $0.05 \mathrm{~nm}$, with the transformation from base e to base 10 (Finlayson-Pitts and Pitts, 2000), it follows for band $1 k_{\mathrm{w} 1}=28.5(\mathrm{~atm} \text { at } 298 \mathrm{~K})^{-1} \mathrm{~cm}^{-1}$ and for band 2 $k_{\mathrm{w} 2}=49.5(\mathrm{~atm} \text { at } 298 \mathrm{~K})^{-1} \mathrm{~cm}^{-1}$ (Fig. 2). Ogawa and Ogawa (1975) $)^{1}$ reported about absorption coefficients for oxygen with a spectral resolution of about $0.1 \mathrm{~nm}$. According to this study, the oxygen absorption coefficient in band 1 is $k_{01}=19.9(\text { atm at } 298 \mathrm{~K})^{-1} \mathrm{~cm}^{-1}$ (nearly identical with the water vapour absorption) and for band $2: k_{02}=32.5$ (atm at $298 \mathrm{~K})^{-1} \mathrm{~cm}^{-1}$. Furthermore, in this spectral range the absorption maximum of ozone with about $k_{031} \approx 83$ (atm at $298 \mathrm{~K})^{-1} \mathrm{~cm}^{-1}$ and $k_{032} \approx 170(\text { atm at } 298 \mathrm{~K})^{-1} \mathrm{~cm}^{-1}$ is based on a spectral resolution of about 0.6 to $0.8 \mathrm{~nm}$ (Ackerman, 1971) ${ }^{1}$, but the ozone concentration is many times lower than the oxygen and water vapour concentrations.

Because the signal of band 1 is more than $20 \%$ lower than that of band 2 due to the lower intensity (Fig. 1) and the smaller width of the band, an estimate of the error resulting from the use of oxygen as a proxy for the calibration can be easily made using the ratio of the water vapour and oxygen absorption in the band 2, which is $k_{\mathrm{w} 2} / k_{\mathrm{o} 2}=1.5$. With a typical ratio of the densities for oxygen of $240 \mathrm{~g} \mathrm{~m}^{-3}$ and water vapour of $10 \mathrm{~g} \mathrm{~m}^{-3}$, it follows that the cross sensitivity of water vapour is less than $10 \%$, and for low water vapour pressure, which is the typical application range of krypton hygrometers, even lower than $5 \%$.

For the calibration with variable path length, the simplification of Eq. (2) given in Eq. (3) cannot be used to determine an effective absorption coefficient. But the assumption will be made that Eq. (2) can be written analogous to Eq. (3) with only one output signal as a combination of both spectral lines in the form

$I=I_{0} \exp \left\{-x\left[\frac{f}{1-f}\left(k_{\mathrm{wl}} \rho_{\mathrm{wl}}+k_{\mathrm{o} 1} \rho_{\mathrm{o} 1}\right)+k_{\mathrm{w} 2} \rho_{\mathrm{w} 2}+k_{\mathrm{o} 2} \rho_{\mathrm{o} 2}\right]\right\}$.

As given above, the most significant absorption is in band 2 due to oxygen, and Eq. (5) can be written in the following form

$I=I_{0} \exp \left(-x k_{\mathrm{o}} \rho_{\mathrm{o}}\right)$

by accepting an error of the calibration of about $10 \%$, which can be reduced for calibrations in different humidity classes. The calibration procedure with variable path length is based on the following assumptions:

- The calibration coefficient can be determined by variation the path length with constant gas concentrations.

\footnotetext{
${ }^{1}$ Data from "MPI-Mainz-UV-VIS Spectral Atlas of Gaseous Molecules" (http://www.atmosphere.mpg.de/enid/2295)
}

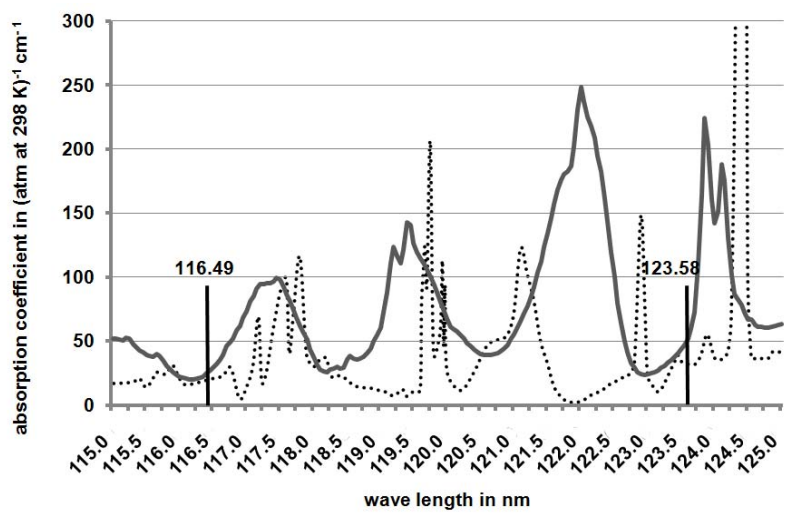

Fig. 2. Absorption coefficient of water vapour in the relevant spectral lines with the data given by Mota et al. (2005) and for oxygen (dotted line) with data given by Ogava and Ogava (1975) and transformed from base e to base 10 (Finlayson-Pitts and Pitts, 2000).

- The intensity of the krypton lamp has the same influence on the water vapour absorption and on the oxygen absorption; therefore, it is possible to use oxygen for the determination of the calibration of the lamp for the case when the oxygen absorption is much larger than the water vapour absorption due to the higher density.

- If a humidity calibration of the krypton hygrometer (e.g. from the producer) and an oxygen calibration (from the calibration device with variable path length) are available for the same time, the calibration coefficient during the lifetime of the krypton hygrometer can be controlled by the oxygen calibration alone.

According to Eq. (4), it follows for the oxygen calibration that

$\ln (V)=\ln \left(V_{0}\right)-\rho_{\mathrm{o}} \cdot x \cdot K_{\mathrm{o}}$.

By comparison of the coefficient $K_{\mathrm{o}}$ of the oxygen calibration with the value of $K_{\mathrm{o}}$ from the first oxygen calibration (for which also a humidity calibration is available), the coefficient of the humidity calibration $K_{\mathrm{w}}$ can be corrected. Because of the cross-sensitivity to humidity of the oxygen calibration, the error is less than $10 \%$, which is much less than the change of the intensity of the krypton lamp within one year. According to Tanner et al. (1993), the effect of oxygen fluctuations due to pressure fluctuations is nearly negligible in comparison to the water vapour fluctuation.

Finally, with the calibration coefficient for oxygen $K_{\mathrm{o}}$ and its change with time, the change of the calibration coefficient for water vapour $K_{\mathrm{w}}$ can also be recalculated according to

$K_{\text {w-new }}=K_{\text {w-old }} \frac{K_{\text {o-new }}}{K_{\text {o-old }}}$.

It is important that for a new instrument the water vapour calibration from Campbell Scientific, Inc. and an oxygen calibration performed with the described calibration device are 


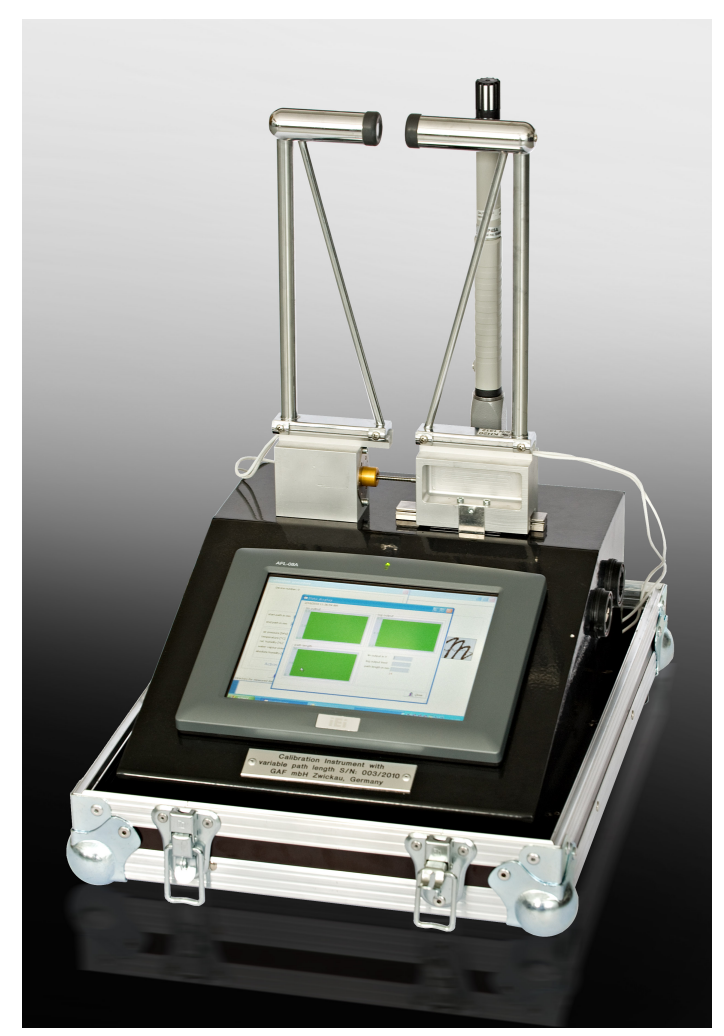

Fig. 3. Calibration system with krypton hygrometer, stepping motor, HMP45A temperature-humidity sensor and touchpanel PC, the switch, fuses and connector for hygrometer at the left side and USB as well as network connector at the right side (photograph: B. Mast)

available. Otherwise a water vapour calibration for constant oxygen concentration must be repeated using a variable dewpoint chamber.

\section{Calibration device and calibration procedure}

\subsection{Calibration device}

The calibration device is designed as one unit containing a stepper motor system, PC and humidity sensor. The system is shown in Fig. 3. A schematic diagram of the device is given in Fig. 4. The standard accessory of the device is a HMP45A temperature-humidity sensor (or updated type HMP 155), which measures temperature and relative humidity. It is also possible to use other humidity devices, and the calibration device therefore includes a humidity calculator. Together with an external pressure sensor, these data are necessary to calculate the air density and therefore the oxygen partial density used in Eq. (7). Relevant textbooks can be consulted for the calculations. The krypton hygrometer is connected with the standard connection cables to the calibration unit. For calibration the KH20 must be fixed on the calibration unit. To determine the exact path length, the minimal

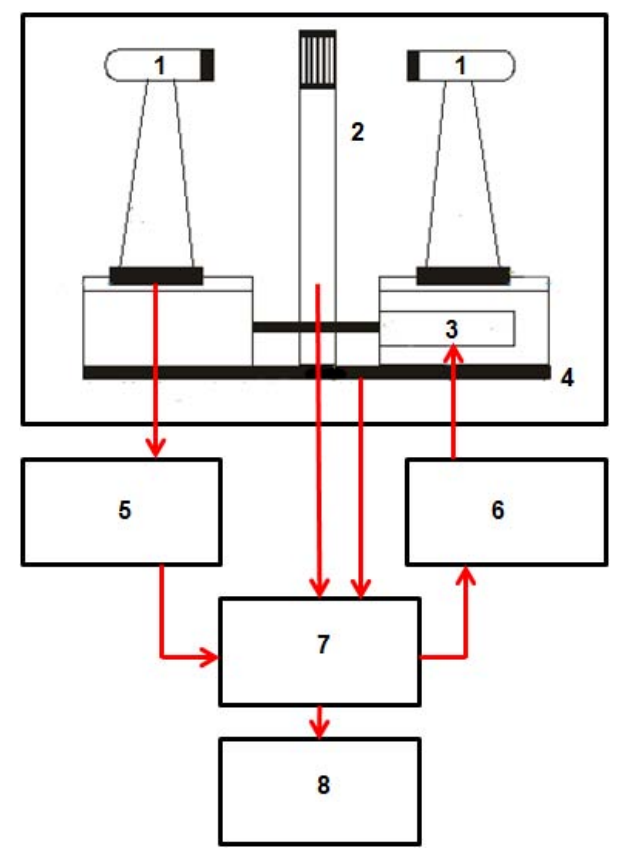

Fig. 4. Block schema of the krypton hygrometer calibration device: 1. KH20, 2. HMP45A, 3. stepper motor, 4. positioning unit with linear slide, 5. amplifier (part of KH20), 6. driver, 7. multifunctionIO, 8: touchpanel PC, the power supply units are not shown.

path length of calibration can be set with distance elements $(2,4$ and $10 \mathrm{~mm})$. It is possible to start the calibration after a delay time of $100 \mathrm{~s}$ which allows the operator to leave the calibration location. The calibration (Fig. 5) can be followed on a screen. After completion of the calibration, the calibration data together with the previous calibration will be shown and written into a file. Furthermore, a calibration protocol will be created similar to the original one. More detail about the device and the handling are given by Foken and Falke (2010).

\subsection{Calibration procedure}

The calibration procedure follows the calibration protocol of the krypton hygrometer KH20 by Campbell Scientific, Inc., with the exception that instead of different absolute humidities, the path length is changed. The output signal must be measured for about 15-20 different path lengths in order to receive a sufficient number of calibration points. It is assumed that the most exact points of the calibration are in the centre of the range of calibration used. The regression according to Eq. (7) starts with a minimum of 3-5 measuring points $[\ln V, x]$ for a given air density. Measuring points will be added to the regression as long as the linearity is given according to the test parameters of the calibration given in Table 1. Because of the non-ideal log-linear response characteristics of the krypton hygrometer, the range and the accuracy of the log-linear approximation (which is the calibration used in most of the turbulence calculation programmes) 
Table 1. Regression settings.

\begin{tabular}{lll}
\hline Regression setting & $\begin{array}{l}\text { Laboratory } \\
\text { calibration }\end{array}$ & $\begin{array}{l}\text { Outdoor } \\
\text { calibration }\end{array}$ \\
\hline Minimum permitted correlation coefficient for about 8 data points in the linear range & 0.995 & 0.990 \\
Maximum permitted difference of data from the regression line in $\ln (\mathrm{m} V)$ & 0.1 & 0.2 \\
Allowed deviation of the calibration coefficient from the prior calibration in \%/100\% & 0.05 & 0.1 \\
\hline
\end{tabular}

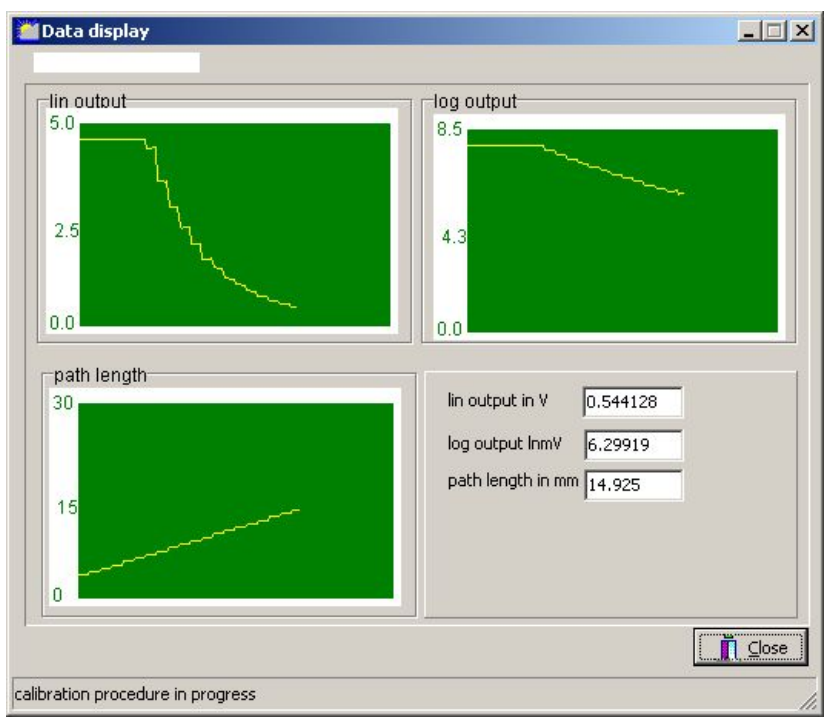

Fig. 5. Screen of the calibration control with the linear (top left panel) and logarithmic (top right panel, comparable with Fig. 6) output signals and the steps of the changing path length (bottom left panel).

is responsible for the accuracy of the calibration. There are two calibration modes: indoor and outdoor calibration. The indoor calibration using typical outdoor conditions (temperature, humidity) is recommended. It is strongly recommended to perform the outdoor calibration under moderate turbulent conditions in the morning or late afternoon hours. If the regression is not well correlated or is not linear, the calibration must be repeated. An example of the calibration on the control screen is given in Fig. 5, and of the calibration curve in Fig. 6. Here, the regression in the linear part of the calibration, which is identical with the linear part of the response characteristics of the krypton hygrometer, is shown between the logarithm of the output signal and the path length according to Eq. (7). The partial densities of oxygen and water vapour should be constant during the calibration. The error of the calibration method is mainly related to the accuracy of the linear approximation. Therefore, it is recommended that at least three calibrations be made within a short time period. The calibrations typically differ by less than $5 \%$ (Table 1). This includes the error of the linearization and influences of pressure and humidity fluctuations during the calibration. Reasons for miss-calibrations can be excessively

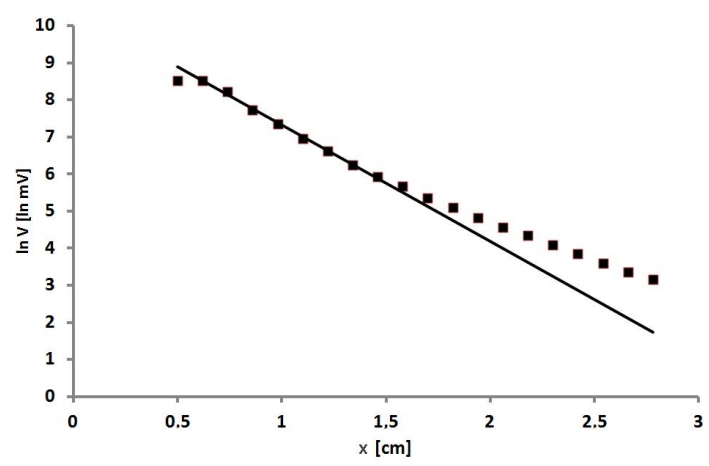

Fig. 6. Example of a calibration diagram, with the measuring points (squares) of the logarithmical output signal in relation to the path length $x$ for nearly constant partial density of water vapour and oxygen (note that this appears similar to the original calibration curve from Campbell Scientific, Inc., except that there the abscissa represents the absolute humidity for constant path length), and the regression line in the nearly log-linear part of the response function, device KH20 No. 1649, Zwickau, 14 July 2011, $\rho_{\mathrm{W}}=7.8 \mathrm{~g} \mathrm{~m}^{-3}$, $\rho_{\mathrm{O}}=241 \mathrm{~g} \mathrm{~m}^{-3}$.

large pressure or humidity fluctuations (outdoor), or a nonsteady state environment (indoor), which allows no log-linear approximation. Other sources of errors, like the temperature and humidity effect on the air density, are negligible because they are much lower than $1 \%$. For the example given in Fig. 6, the cross sensitivity due to water vapour is $4.9 \%$ according to the calculation given in Sect. 2.2. Normally, the absolute humidity $\rho_{\mathrm{w}}$ has no daily cycle like the relative humidity, and significant changes occur only with a change of the air masses. Applying different calibrations for different classes of absolute humidity can reduce the cross sensitivity. The cross sensitivity is even smaller for the typical application of krypton hygrometers under low water vapour concentrations. The error - which is therefore mainly related to the degree to which it is possible to linearize the log-linear calibration - is given above as typically less than $5 \%$.

With the water vapour and oxygen calibration for a new device the determined oxygen calibration will be recalculated into a water vapour calibration with Eq. (8). The first oxygen calibration should be done within the few weeks for which Campbell Scientific, Inc. guarantees the constancy of the calibration coefficient. It is important to clean the window before the calibration and to compare with the 
Table 2. Results of calibrations of the Krypton Hygrometer no. 1649 (produced 2009).

\begin{tabular}{lccc}
\hline Date/Place & $\begin{array}{c}\text { Oxygen density } \\
\text { in } \mathrm{g} \mathrm{m}^{-3}\end{array}$ & $\begin{array}{c}K_{\mathrm{W}}^{*} \\
\text { in } \ln (\mathrm{mV}) \mathrm{m}^{3} \mathrm{~g}^{-1} \mathrm{~cm}^{-1} \\
\text { for constant oxygen } \\
\text { concentration }\end{array}$ & $\begin{array}{c}K_{\mathrm{O}}^{*} \\
\text { in } \ln (\mathrm{mV}) \mathrm{m}^{3} \mathrm{~g}^{-1} \mathrm{~cm}^{-1} \\
\text { for constant water } \\
\text { vapour concentration }\end{array}$ \\
\hline Campbell Scientific, Inc., Logan UT, 1340 m a.s.1. & & -0.1573 & -13.607 \\
Bayreuth, Germany, 340 m a.s.1., 8 May 2009 & 250.43 & $-0.1573 / 0.79$ & -17.223 \\
NamCo, China, 4730 m a.s.l., 25 June 2009 & 144.90 & $-0.1573 / 0.67$ & -20.231 \\
NamCo, China, 4730 m a.s.1., 5 July 2010 & 145,51 & - & \\
\hline
\end{tabular}

${ }^{*} K_{\mathrm{W}}$ and $K_{\mathrm{O}}$ are the calibration coefficients, the sensitivity is the reciprocal value.

Campbell Scientific, Inc. calibration for clean windows, because "scaled window" is not a definite state. The programme compares the calibration with the previous calibration of the same device. If the calibration is within 5 or $10 \%$ of the chosen path length or optimal path length (Table 1), both calibrations differ only within typical errors and no change of the calibration coefficient is necessary.

The results of the calibration are the parameters for a given measuring path length $x[\mathrm{~cm}]$ : constant $V_{0}$ in $[\ln (\mathrm{mV})]$, coefficient $K_{\mathrm{W}}$ in $\left[\ln (\mathrm{mV}) \mathrm{m}^{3} \mathrm{~g}^{-1} \mathrm{~cm}^{-1}\right]$, and the coefficient $x K_{\mathrm{W}}$ in $\left[\ln (\mathrm{mV}) \mathrm{m}^{3} \mathrm{~g}^{-1}\right]$ is also given.

The optimal path length is in the centre of the linear calibration range. This parameter should help to inform the researcher as to whether the chosen path length was optimal according to the operational environment.

\subsection{Improvement of the calibration}

A prototype of the device was successfully tested during several experiments, and the described version during both laboratory measurements at Bayreuth (Germany) and two expeditions on the Tibetan Plateau. The calibration in Bayreuth for a new krypton hygrometer was equal to that of Campbell Scientific, Inc., and on the Tibetan Plateau the data obtained after calibration followed a reference device well. The calibration coefficients found are given in Table 2. A further test under winter conditions (water vapour density about 1$2 \mathrm{~g} \mathrm{~m}^{-3}$ ) showed a lower sensitivity and the necessity to increase the path length. These results will be published separately together with the application to the measurement of turbulent water vapour fluxes.

It can be seen that there is a significant change of the calibration characteristics - about $20 \%$ - after the installation on the Tibetan Plateau and an additional change of more than $10 \%$ within one year. This is much larger than the accuracy of the method of about $5 \%$ and the difference of the calibrations by Campbell Scientific, Inc., with clean and scaled windows, of about $1-2 \%$. For shorter periods of some weeks in the same environment, the calibration differs only within the above given range of accuracy of approximately $5 \%$ (Table 1). This means that the krypton hygrometer is, for typical measuring periods of some weeks with uniform environmental conditions, quite stable with no drift. None of the parts of the calibration device itself (stepper motor, amplifier, standard meteorological sensors) have errors or drifts of a size that would affect the calibration results.

\section{Conclusions}

The Campbell Scientific, Inc. calibration protocol includes calibrations for clean and scaled windows and different ranges of the humidity. All Campbell Scientific, Inc. devices are probably calibrated at an altitude of more than $1000 \mathrm{~m}$ a.s.l. with a lower oxygen concentration than that used in most regions of the world. Therefore, a control of the influence of the oxygen concentration and the conditions of the windows are necessary. With the in-situ calibration (calibration with variable path length) this problem can be overcome, but the calibration can then only be used for the calibration site and must be repeated if the device is used under different conditions or if the windows are scaled.

The application of the calibration device is useful if the windows of the krypton hygrometer are scaled and, of course, for low humidity conditions due to very low temperatures or high altitudes. The calibration procedure takes only a few minutes, but the installation and de-installation of the hygrometer on the device needs some time and care, resulting in an interruption of the measuring programme of about one hour. The final recommendation is to use IR-hygrometers for humidity fluctuation measurements under most of the environmental conditions to measure the latent heat flux, but for the described extreme conditions the krypton hygrometer together with the proposed calibration device - is a useful tool for increasing the accuracy. The application of the calibration device is probably less cost intensive for experiments at remote places than the application of humidity chambers.

Acknowledgements. In memory of Bertrand Tanner (Campbell Scientific, Inc.), who made many efforts to develop the krypton hygrometer and other turbulence measuring techniques.

Edited by: D. Toohey 


\section{References}

Ackerman, M.: UV-solar radiation related to mesospheric processes, in: Mesospheric Models and Related Experiments, edited by: Fiocco, G., D. Reidel Publishing Company, Dordrecht, 149159, 1971.

Buck, A. L.: Development of an improved Lyman-alpha hygrometer, Atmos. Technol., 2, 213-240, 1973.

Buck, A. L.: The variable-path Lyman-alpha hygrometer and its operating characteristics, B. Am. Meteorol. Soc., 57, 1113-1118, 1976.

Campbell, G. S. and Tanner, B. D.: A Krypton hygrometer for measurement of atmospheric water vapour concentrations, in: Moisture and Humidity, ISA, Research Triangle Park, 609-614, 1985.

Finlayson-Pitts, B. J. and Pitts, J. N.: Chemistry of the Upper and Lower Atmosphere, Academic Press, San Diego, 963 pp., 2000.

Foken, T. and Falke, H.: Documentation and instruction manual for the Krypton hygrometer calibration instrument, Arbeitsergebn., Univ. Bayreuth, Abt. Mikrometeorol., 42, 20 (update 2011), 2010.

Foken, T., Dlugi, R., and Kramm, G.: On the determination of dry deposition and emission of gaseous compounds at the biosphereatmosphere interface, Meteorol. Z., 4, 91-118, 1995.

Foken, T., Buck, A. L., Nye, R. A., and Horn, R. D.: A Lyman-alpha hygrometer with variable path length, J. Atmos. Ocean. Tech., 15, 211-214, 1998.

Johns, J. W. C.: The absorption of radiation by water vapor, in: Humidity and moisture, measurement and control in science and industry, vol. 1, Principles and Methods of Measuring Humidity in Gases, edited by: Ruskin, R. E., Reinhold, New York, 417-427, 1965.
Kretschmer, S. I. and Karpovitsch, J. V.: Maloinercionnyj ultrafioletovyj vlagometer (Sensitive ultraviolet hygrometer), Izv. AN SSSR, Fiz. Atm. i Okeana, 9, 642-645, 1973.

Martini, L., Stark, B., and Hunsalz, G.: Elektronisches LymanAlpha-Feuchtigkeitsmessgerät, Meteorol. Z., 23, 313-322, 1973.

Mota, R., Parafita, R., Giuliani, A., Hubin-Franskin, M. J., Lourenço, J. M. C., Garcia, G., Hoffmann, S. V., Mason, M. J., Ribeiro, P. A., Raposo, M., and Limão-Vieira, P.: Water VUV electronic state spectroscopy by synchrotron radiation, Chem. Phys. Lett., 416, 152-159, 2005.

Ogawa, S. and Ogawa, M.: Absorption cross sections of O2(a1 $\Delta \mathrm{g})$ and $\mathrm{O} 2(\mathrm{X} 3 \Sigma \mathrm{g}-)$ in the region from 1087 to $1700 \AA$, Can. J. Phys., 53, 1845-1852, 1975.

Tanner, B. D., Swiatek, E., and Greene, J. P.: Density fluctuations and use of the krypton hygrometer in surface flux measurements, in: Management of Irrigation and Drainage Systems: Integrated Perspectives, edited by: Allen, R. G., American Society of Civil Engineers, New York, NY, 945-952, 1993.

Tillman, J. E.: Water vapor density measurements utilizing the absorption of vacuum ultraviolet and infrared radiation, in: Humidity and moisture, Measurement and control in Science and Industry, vol. 1, Principles and methods of measuring humidity in gases, edited by: Ruskin, R. E., Reinhold, New York, 428-433, 1965.

van Dijk, A., Kohsiek, W., and DeBruin, H. A. R.: Oxygen sensitivity of krypton and Lyman-alpha hygrometers, J. Atmos. Ocean. Tech., 20, 143-151, 2003.

Watanabe, K. and Zelikoff, M.: Absorption coefficient of water vapor in the vacuum ultraviolet, J. Opt. Soc. Am., 43, 753-754, 1953. 